\title{
Validation of the Radimer/Cornell food insecurity measure in rural Kilimanjaro, Tanzania
}

\author{
Germana H Leyna ${ }^{1,2, *}$, Elia J Mmbaga ${ }^{1,3}$, Kagoma S Mnyika ${ }^{1}$ and Knut-Inge Klepp ${ }^{2}$ \\ 'Department of Epidemiology and Biostatistics, Muhimbili University College of Health Sciences, Dar es Salaam, \\ Tanzania: ${ }^{2}$ Department of Nutrition, Faculty of Medicine, University of Oslo, PO Box 1046 Blindern, N-0316 \\ Oslo, Norway: ${ }^{3}$ Department of General Practice and Community Medicine, University of Oslo, Oslo, Norway
}

Submitted 11 March 2007: Accepted 9 September 2007: First published online 16 November 2007

\begin{abstract}
Objective: The objective of the present study was to assess the construct validity, criterion-related validity and internal consistency of the Radimer/Cornell food insecurity measure for use in rural Tanzania.

Design: A cross-sectional community-based survey was conducted from March to May 2005. Key adaptations to the nine-item Radimer/Cornell items included translation to Swahili, replacing the term 'balanced diet' with 'full meal' and constructing the items as questions rather than statements. Factor analysis and Cronbach's alpha were used to assess validity and reliability, respectively.

Setting: Rural Kilimanjaro, Tanzania.

Subjects: Analysis was restricted to data from 530 women aged 15-44 years who had children under 5 years old.

Results: Principal component factor analysis revealed a two-factor solution: (1) altered eating pattern at household level and (2) altered eating pattern at child level. The two factors accounted for $66.2 \%$ of the total variance. The subscales developed had good reliability. Internal consistency of the scales was 0.853 and 0.784 for food insecurity at household level and food insecurity at child level, respectively. Only $14.0 \%$ of the women reported to be food-secure and $86.0 \%$ reported some kind of food insecurity. The Radimer/Cornell food insecurity measure showed significant associations with selected sociodemographic factors in the expected directions. There was also an association with the NHANES III (Third National Health and Nutrition Examination Survey) and CCHIP (Community Childhood Hunger Identification Project) indicators.

Conclusion: Our findings suggest that the adapted Radimer/Cornell measure may have some utility in assessing food insecurity in settings like rural Tanzania.
\end{abstract}

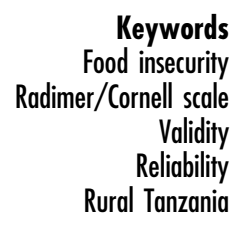

The current number of people suffering from undernutrition globally is estimated at 852 million, with a third of these in sub-Saharan Africa alone ${ }^{(1)}$. Although some of these countries report to have adequate food at the national level, this does not guarantee food security at the household level. Yet most of the studies using household food insecurity measures have been conducted in developed countries where the prevalence of food insecurity is relatively low ${ }^{(2,3)}$.

Food security is a complex issue; it is defined as 'a state where all people, at all times, have physical and economic access to sufficient, safe and nutritious food to meet their dietary needs and food preferences for an active and healthy life ${ }^{(4)}$. The demand for an instrument to measure food insecurity in a simple but valid and reliable manner is high. It is needed by policy-makers, researchers and programme officers trying to target, monitor and evaluate interventions and policy measures aimed at improving food security. However, before approaching the subject of prevalence and determinants of food insecurity in developing countries, the problem of measurement must be adequately addressed.

Most developing countries still rely on aggregate data using food balance sheets and early-warning systems that do not necessarily provide adequate information on household-level food security ${ }^{(5)}$. The use of household perceptions of food needs and coping strategies as an alternative indicator in measuring food insecurity has proved to be a good and cost-effective method ${ }^{(6)}$. Radimer and colleagues have utilised these food insecurity perceptions by developing a 'hunger index' through qualitative interviews with women from low-income households $^{(7)}$. These indices have proved to be valid and reliable in the context for which they were developed ${ }^{(7,8)}$ 
and also in some developing countries where they have been used ${ }^{(9-12)}$. However, there are indications that validated measurements may vary in their performance across different population groups and cultures ${ }^{(13-15)}$. Also, cut-off points used to identify 'at-risk' or 'affected' households can result in inaccurate estimates without proper renaming, as scores that are numerically identical may have different severity meanings across cultures ${ }^{(15)}$.

The objective of the present study was to assess the construct validity, criterion-related validity and internal consistency of the Radimer/Cornell food insecurity measure for use in rural Kilimanjaro, Tanzania.

\section{Methods}

\section{Location}

A more detailed description of the area has been provided elsewhere $^{(16,17)}$. In brief, Oria village is part of Kahe Ward in Moshi rural district, located at the foot of Mount Kilimanjaro about $30 \mathrm{~km}$ south of the regional administrative capital, Moshi. The village has a weekly market where a wide variety of commodities are sold. The market is used by local and neighbouring villagers as well as business persons from Moshi.

\section{Subjects}

Of the 2093 inhabitants aged 15-44 years who were invited to participate in the study, $1528(73.0 \%)$ agreed. Lack of permanent address in the village was the only exclusion criterion. The present analysis is restricted to data from women aged 15-44 years with children under 5 years old $(n=530)$. The population mainly consisted of peasants who grow maize, paddy and vegetables, petty businessmen selling agricultural products and fish, and a few employed individuals. Most of the villagers were also involved in farm and non-farm labour to supplement their income.

\section{Instrument adaptation}

A nine-item Radimer/Cornell food insecurity measure (Table 1) was translated to Swahili by two of the principal authors. All items were phrased as questions, with responses categorised as 'never', 'sometimes' or 'often'. The phrase 'balanced meal' for the children (Q7) was translated to 'mlo kamili meaning 'full meal'. This is interpreted to mean a meal with meat, starch, vegetables and fruit, and confirms with local traditional knowledge $^{(18)}$. The translated instrument was then subjected to a question-by-question review by the authors and 10 research assistants with good knowledge of the research area and experience in nutrition counselling at local maternal and child health clinics. Each item was examined to ensure face validity in the local context and a transfer of conceptual meaning in Swahili. The revised
Table 1 Survey items used to validate the Radimer/Cornell food insecurity measure in rural Kilimanjaro, Tanzania

1. Do you worry that your family may run out of food before you have money to buy again?

2. Do you worry about where the next day's food is going to come from?

3. Has your family ever eaten the same type of food for several days consecutively because you did not have enough money to buy different food?

4. Can you afford to eat the way you think you should?*

5. Have you often been hungry but don't eat because you can't afford enough food?

6. Have you ever eaten less than you wanted because you don't have enough money to buy food?

7. Do you not have enough money to buy healthy and nutritious or balanced food for your children?

8. Have your children (according to you) not had enough to eat because you do not have enough money to buy food?

9. Do you know that your children are hungry sometimes but you just do not have enough money to buy food?

*Question was excluded from the analysis.

instrument was then pre-tested in a rural community with similar characteristics to the study population. A convenience sample of 150 participants was used.

\section{Data collection procedure}

A cross-sectional survey was conducted during the preharvest (rainy) season from March to May 2005. Eligible participants were identified through house-to-house registration and counter-checked with existing village census lists. Interviews were conducted with each respondent near their home by a team of trained research assistants under supervision of one of the principal authors. A revised version of a previously used questionnaire was used to collect information on sociodemographic characteristics. The questionnaire included the Third National Health and Nutrition Examination Survey (NHANES III) food sufficiency question and a question on woman's meal skipping from the Community Childhood Hunger Identification Project (CCHIP). The food sufficiency question administered was "Which of the following statements best describes the amount of food eaten in your household: (1) have enough to eat; (2) sometimes have enough to eat; and (3) often do not have enough to eat?'

\section{Assessment of validity}

Three aspects of validity of the Radimer/Cornell measure were assessed: (1) construct validity using principal component factor analysis; (2) internal consistency (precision) using Cronbach's alpha; and (3) criterion-related validity (accuracy) by comparing the developed Radimer/ Cornell measure with sociodemographic factors and the NHANES and CCHIP questions. In the absence of a criterion measure (gold standard), accuracy can be assessed by comparing the food insecurity measure with comparison measures such as determinants, consequences of food insecurity or other measures of food insecurity ${ }^{(19)}$. 
This analytic strategy has been well described and used by Frongillo ${ }^{(20,21)}$.

The study received research ethical approval from the Tanzanian Ethical Committee of the Ministry of Health and from the Norwegian Committee for Medical Research Ethics. All participants provided written or a thumb print informed consent. Individual health education was given to all participants to correct any misconceptions concerning the study and all households in the village were provided with a small food package irrespective of their participation status.

\section{Data analysis}

Frequency distribution of all Radimer/Cornell food insecurity items was done for descriptive purposes. The food insecurity items were then subjected to principal component factor analysis. Two- to four-factor solutions were explored and followed up by oblique rotation to assist in the interpretation. Factors with an eigenvalue of approximately one or more were retained. Subscales were developed using items that consistently grouped together and had factor loading with an absolute value of 0.4 or more. These items were considered to reflect the same construct. Internal consistency of the developed scales was assessed using Cronbach's alpha. A scale with an $\alpha$ coefficient of 0.7 or higher was considered reliable ${ }^{(22)}$. The question on 'affording to eat the way you think you should' (Q4, Table 1) was omitted from the analysis due to: (1) poor correlation with other Radimer/Cornell food insecurity items $(r<0 \cdot 2)$; (2) when removed the internal consistency of the scale improved from Cronbach's $\alpha=0.802$ to 0.853 ; and (3) the percentage of affirmative response $(75.7 \%)$ was higher than for other items. The $\chi^{2}$ test for trend in proportions was run to compare sociodemographic characteristics and the NHANES III and CCHIP food insecurity indicators across the Radimer/ Cornell food insecurity categories. A two-tailed $P$ value of $<0.05$ was considered significant. All analyses were carried out using SPSS version 12.0 (SPSS Inc., Chicago, IL, USA).

\section{Results}

\section{Sample characteristics}

Demographic characteristics of the women in the sample are summarised in Table 2. The mean age of the respondents was 28.0 (standard deviation 6.4) years. Most of the women $(78.5 \%$; 416/530) were married or cohabiting and in a monogamous relationship (83.2\%; 346/ 416). About $81.0 \%(427 / 530)$ had primary education and a majority $(83 \cdot 2 \% ; 441 / 530)$ were peasants.

\section{Radimer/Cornell food insecurity items}

Affirmative responses to the eight food insecurity items ranged from $43.2 \%$ to $68.3 \%$. The most frequently endorsed food insecurity item was Q3, 'Has your family ever eaten the same type of food for several days consecutively because you did not have enough money to buy different food?', as noted in Table 3. The least endorsed food insecurity item was Q4, 'Have you often been hungry but do not eat because you can not afford

Table 2 Sample characteristics of women with children under 5 years old in rural Kilimanjaro, Tanzania

\begin{tabular}{|c|c|c|}
\hline Variable & $n$ & $\%$ \\
\hline Age (years) ${ }^{\star}$ & 530 & $28.0(6.4)$ \\
\hline \multicolumn{3}{|l|}{ Marital status } \\
\hline Single & 46 & 8.7 \\
\hline Married/cohabiting & 416 & 78.5 \\
\hline Divorced/separated/widowed & 68 & $12 \cdot 8$ \\
\hline \multicolumn{3}{|l|}{ Education } \\
\hline None & 62 & 11.7 \\
\hline Primary education & 427 & 80.6 \\
\hline Secondary education and above & 41 & 7.7 \\
\hline \multicolumn{3}{|l|}{ Occupation } \\
\hline Peasant & 441 & 83.2 \\
\hline Others & 89 & $16 \cdot 8$ \\
\hline \multicolumn{3}{|l|}{ Religion } \\
\hline Christian & 279 & 52.6 \\
\hline Moslem & 251 & 47.4 \\
\hline
\end{tabular}

*Mean (standard deviation).

Table 3 Distribution of affirmative responses and rotated factor loadings of the Radimer/Cornell food insecurity items as assessed in rural Kilimanjaro, Tanzania

\begin{tabular}{|c|c|c|}
\hline Item & $\% *$ & Factor loadings \\
\hline Household level & & Factor $1+$ \\
\hline Do you worry that your family may run out of food before you have money to buy again? & $56 \cdot 8$ & 0.857 \\
\hline Do you worry about where the next day's food is going to come from? & $55 \cdot 8$ & 0.863 \\
\hline $\begin{array}{l}\text { Has your family ever eaten the same type of food for several days consecutively because you did not have } \\
\text { enough money to buy different food? }\end{array}$ & 68.3 & 0.746 \\
\hline Have you often been hungry but don't eat because you can't afford enough food? & $43 \cdot 2$ & 0.711 \\
\hline Have you ever eaten less than you wanted because you don't have enough money to buy food? & $55 \cdot 6$ & 0.665 \\
\hline Child level & & Factor $2 \ddagger$ \\
\hline Do you not have enough money to buy healthy and nutritious or balanced food for your children? & 51.5 & 0.742 \\
\hline $\begin{array}{l}\text { Have your children (according to you) not had enough to eat because you do not have enough money to } \\
\text { buy food? }\end{array}$ & 54.5 & 0.761 \\
\hline Do you know that your children are hungry sometimes but you just do not have enough money to buy food? & $60 \cdot 4$ & 0.891 \\
\hline
\end{tabular}

*Responding 'sometimes' or 'often' to the Radimer/Cornell food insecurity items.

tFactor 1 explained $55.2 \%$ of the total variance.

¥Factor 2 explained $11.0 \%$ of the total variance. 
enough food?' About $82.0 \%$ (432/530) of the women responded positively to one or more of the food insecurity items at the household level and 71.1\% (377/530) responded that their children had experienced one or more of the food insecurity items at the child level. Only $14.0 \%(74 / 530)$ of the women responded 'never' to all questions and were characterised as food-secure.

\section{Construct validity}

The rotated principal component factor analysis of the Radimer/Cornell food insecurity items revealed two factors: (1) altered eating pattern at household level and (2) altered eating pattern at child level (Table 3). Five items loaded onto the first factor with loadings ranging from 0.67 to 0.86 . The remaining three items came together as the second factor with loadings between 0.74 and 0.89 . The total variance explained by these two factors was $66.2 \%$. The first factor extracted explained $55.2 \%$ of the total variance and the second factor accounted for $11.0 \%$ of the total variance.

\section{Internal consistency}

The subscales had good reliability. Cronbach's $\alpha$ for the household subscale was 0.853 ( $n=5$ items) and for the child subscale 0.784 ( $n=3$ items).

\section{Criterion-related validity}

Criterion-related validity was assessed by creating the Radimer/Cornell food insecurity measure and investigating its relationship to sociodemographic factors and the NHANES III and CCHIP food insecurity indicators. The measure was created by assigning women to three exclusive groups: (1) all women responding 'never' to all food insecurity items were categorised as food-secure (14.0\%); (2) all women responding 'sometimes' or 'often' to the household factors but not any of the child-level factors were categorised as food-insecure at household level (17.4\%; 92/530); and (3) all women who responded 'sometimes' or 'often' to the child-level factors were categorised as food-insecure at child level (68.7\%; 364/530).

Table 4 depicts the relationship between sociodemographic factors, the NHANES III indicator, the CCHIP indicator and the Radimer/Cornell food insecurity measure. Food insecurity status was strongly associated with most of the sociodemographic characteristics. Age and marital status were significantly and positively correlated with food insecurity. Women who were categorised as food-insecure at the child level were more likely to be older ( $\geq 33$ years) and separated, divorced or widowed. On the other hand, education and occupation were significantly and negatively correlated with food insecurity, whereby as food insecurity status worsened

Table 4 Sociodemographic characteristics of the sample population and other food insecurity indicators by Radimer/Cornell food insecurity status in rural Kilimanjaro, Tanzania

\begin{tabular}{|c|c|c|c|c|c|}
\hline \multirow[b]{2}{*}{ Variable } & \multirow[b]{2}{*}{$n$} & \multirow{2}{*}{$\begin{array}{c}\text { Food-secure } \\
(n=74)\end{array}$} & \multirow{2}{*}{$\begin{array}{c}\begin{array}{c}\text { Food insecurity at } \\
\text { household level }(n=92)\end{array} \\
(\%)\end{array}$} & \multirow{2}{*}{$\begin{array}{c}\begin{array}{c}\text { Food insecurity at } \\
\text { child level }(n=364)\end{array} \\
(\%)\end{array}$} & \multirow[b]{2}{*}{$P$ value } \\
\hline & & & & & \\
\hline \multicolumn{6}{|l|}{ Age } \\
\hline$<24$ years & 155 & $16 \cdot 1$ & 23.2 & $60 \cdot 6$ & \\
\hline $24-27$ years & 115 & $16 \cdot 5$ & $18 \cdot 3$ & 65.2 & \\
\hline $28-32$ years & 128 & 14.8 & $15 \cdot 6$ & 69.5 & \\
\hline$\geq 33$ years & 132 & $8 \cdot 3$ & 11.4 & $80 \cdot 3$ & 0.001 \\
\hline \multicolumn{6}{|l|}{ Marital status } \\
\hline Single & 46 & 13.0 & 19.6 & 67.4 & \\
\hline Married/cohabiting & 416 & 15.4 & $18 \cdot 3$ & $66 \cdot 3$ & \\
\hline Separated/divorced/widowed & 68 & $5 \cdot 9$ & $10 \cdot 3$ & 83.8 & 0.031 \\
\hline \multicolumn{6}{|l|}{ Education } \\
\hline No formal education & 62 & $8 \cdot 1$ & 14.5 & 77.4 & \\
\hline Primary education & 427 & $14 \cdot 1$ & $16 \cdot 6$ & $69 \cdot 3$ & \\
\hline Higher education & 41 & 22.0 & 29.3 & 48.8 & 0.004 \\
\hline \multicolumn{6}{|l|}{ Occupation } \\
\hline Peasant & 441 & 13.6 & $15 \cdot 6$ & 70.7 & \\
\hline Others & 89 & $15 \cdot 7$ & $25 \cdot 8$ & 58.4 & 0.050 \\
\hline \multicolumn{6}{|l|}{ Religion } \\
\hline Christian & 279 & $12 \cdot 2$ & $15 \cdot 8$ & $72 \cdot 0$ & \\
\hline Moslem & 251 & $15 \cdot 9$ & $19 \cdot 1$ & 64.9 & 0.077 \\
\hline \multicolumn{6}{|l|}{$\begin{array}{l}\text { Other food insecurity measures } \\
\text { NHANES IIIt }\end{array}$} \\
\hline Sufficient food & 197 & 31.5 & 23.9 & $44 \cdot 7$ & \\
\hline Insufficient food & 333 & 3.6 & 13.5 & 82.9 & $<0.001$ \\
\hline \multicolumn{6}{|l|}{ CCHIP } \\
\hline Woman does not skip meal & 408 & 17.9 & 19.9 & 62.3 & \\
\hline Woman skips meal & 122 & 0.8 & $9 \cdot 0$ & 90.2 & $<0.001$ \\
\hline
\end{tabular}

${ }^{*} P$ value for $\chi^{2}$ test for trend across food insecurity status.

tFood sufficiency question from the Third National Health and Nutrition Examination Survey.

$¥$ Woman's meal skipping question from the Community Childhood Hunger Identification Project. 
women were more likely to have no formal education and be involved in farming. Overall, the Radimer/Cornell food insecurity measure agreed on $72.3 \%$ (383/530) of the women with the NHANES III indicator and on $36.7 \%$ (194/530) of the women with the CCHIP indicator on the determination of food insecurity status.

\section{Discussion}

The purpose of the present study was to determine the validity and reliability of the Radimer/Cornell food insecurity measure in assessing food insecurity in a rural Tanzanian population. The final adapted scale in this study had eight Radimer/Cornell food insecurity items including the question on food monotony suggested by Kendall et $a l .{ }^{(8)}$. The definition of 'balanced diet' as used by Radimer and other food security tools has been observed to be difficult to use in developing countries ${ }^{(9,23)}$. In this study the term 'balanced food' was conceptualised to reflect local knowledge which is similar to traditional definitions ${ }^{(18)}$.

Principal component factor analysis revealed two factors that identified altered eating patterns at the household and child level. Other studies have found the same factors in addition to two other factors, namely individuallevel factor and qualitative factor. The total variance explained by the two identified factors compares well with other studies ${ }^{(7,8)}$. Moreover, the internal consistency of our subscales is good and similar to others although the child food insecurity subscale is slightly lower ${ }^{(7,8)}$. The inability of our study to identify these other factors may be due to: (1) the use of fewer items addressing quality aspects of food insecurity; (2) the population studied clearly was more vulnerable to food insecurity and thus overrode the qualitative aspects of food insecurity with the quantitative aspects ${ }^{(8,15)}$; and (3) the scale used was primarily developed for a different target population and hence lacked culturally sensitive expressions that related to the other factors. However, the absence of these other factors of food insecurity (qualitative and individual-level factor) in our analysis does not exclude them from existing in the study population.

Food insecurity has been described as a managed process where households and individuals respond actively to the risks they encounter on a daily basis ${ }^{(7)}$. These responses have been noted to follow a general pattern and have led researchers to use them as a 'proxy' for the severity of food insecurity. The two factors identified in our study covered a range of elements of the food insecurity concept. The questions related to presence of anxiety (Q1 and Q2), the quality of food consumed (Q3 and Q7) and a lack of food in quantity (Q4, Q5, Q6 and Q8). The distribution of affirmative responses showed that these experiences were common in this population. However, the pattern of the affirmative responses in our data differed from that in studies conducted elsewhere, where child issues had lower affirmative responses compared with household issues and adult issues ${ }^{(8,9,24)}$. Response patterns of households when confronted with food constraints have been shown to differ across and within cultures ${ }^{(6,15)}$, as well as between households with and without children ${ }^{(13)}$. In addition, the age of children referred to in our study was younger ( $0-5$ years) whereas other studies defined children as between 0 and 18 years $\operatorname{old}^{(7,8)}$. Thus, the pattern of affirmative responses observed suggests the distribution of behaviours adopted during these situations, rather than the degree of severity of food insecurity as observed elsewhere ${ }^{(8,9,24)}$. Further research is needed to identify coping strategies geared towards protecting children that may identify severity of food insecurity in this community.

Approximately $69.0 \%$ of the women were classified as having food insecurity at child level. Women whose children were categorised as food-insecure were also more likely to be from a household considered to be food-insecure. These categories used to describe food insecurity have been suggested by Radimer et al. and reaffirmed by other researchers ${ }^{(8,11,24)}$. Responding 'sometimes' or 'often' to the food insecurity items identifies a household or individual into a certain group of food insecurity status. This hunger index developed by Radimer et al. (and utilised in this study) is conceptually clear, simple and straightforward to identify households in need of intervention. It is practical in developing countries where there is limited skilled manpower to analyse complex data. However, in order to identify those in immediate need of assistance, a different method of categorisation may be appropriate in order to spread out the households. The use of scores or addition of other categories may work as alternatives ${ }^{(9,10)}$.

The selected sociodemographic characteristics showed a significant association with food insecurity status in the expected directions. This finding is consistent with other studies ${ }^{(7-10)}$. In the assessment of food insecurity status, the agreement of the NHANES III indicator was good but poor for the CCHIP indicator. However, both indicators were very good at categorising women who were food-secure. The NHANES III and CCHIP indicators seem to measure less severe food insecurity status which the Radimer/ Cornell measure assesses as food security. This supports the ability of the Radimer/Cornell food insecurity measure to identify households experiencing increasingly severe food insecurity levels in this rural population.

The strength of this study lies in the use of a sample of women from the general population to validate the Radimer/Cornell food insecurity measure in rural Tanzania. The sample provided a broad range of food insecurity experiences to be included in the study. This allowed a modest inference to the external validity of the Radimer/Cornell measure in other rural areas of Kilimanjaro where similar situations may exist ${ }^{(25)}$. Although the adapted Radimer/Cornell measure addressed several of the domains of food insecurity, its use as a generic tool may have limited the scope of food insecurity 
experiences that could be identified within the population. Also, due to lack of a criterion measure ('gold standard') of food insecurity in Tanzania, the accuracy of the Radimer/Cornell tool is debatable. However, the consistent and significant association of the measure to its determinants education and occupation, and the other food insecurity measures in the expected directions, provides reasonable assurance that the measure is functioning as intended. Furthermore, the Radimer/Cornell measure at this stage cannot be used for monitoring purposes as its stability across time was not assessed.

In conclusion, our findings suggest that the adapted Radimer/Cornell measure may have some utility in assessing food insecurity in settings like rural Tanzania. This could be a simple and cheap alternative to provide timely and informative information on food and nutrition needs at the household level. Further research is needed to assess the accuracy of experienced-based measures of food security in Africa and explore culturally sensitive expressions of food insecurity that could be included to improve assessment.

\section{Acknowledgements}

The authors are grateful to the participants for their time and to the hardworking spirit of the research team. This study was funded in part by a grant from the Norwegian Programme for Development, Research and Higher Education (NUFU) and facilitated by the collaborating institutions: Muhimbili University College of Health Sciences, Kilimanjaro Christian Medical College and Centre for Education Development Arusha in Tanzania and the Universities of Oslo and Bergen, Norway. The contribution of each authors are: G.H.L., conception and design of the study, drafting the manuscript, analysis and interpretation of the data; E.J.M., conception and design of the study, analysis, interpretation of the data and substantial revision of the manuscript; K.S.M., analysis, interpretation of the data and substantial revision of the manuscript; K.-I.K., conception and designing of the study, analysis, interpretation of the data and substantial revision of the manuscript. The authors declare that they have no competing interests in whatever form.

\section{References}

1. Food and Agriculture Organization of the United Nations (2004) The State of Food Insecurity in the World 2004. Rome: FAO.

2. Nord M, Andrews M \& Carlson S (2005) Household Food Security in the United States, 2004. Economic Research Report no. ERR11. Washington, DC: US Department of Agriculture.

3. Vozoris NT \& Tarasuk VS (2003) Household food insufficiency is associated with poorer health. J Nutr 133, 120-126.

4. Food and Agriculture Organization of the United Nations (1998) Rome Declaration on World Food Security. World Food Summit 1996. Rome: FAO.
5. Quinn VJ \& Kennedy E (1994) Food security and nutrition monitoring systems in Africa. A review of country experiences and lessons learned. Food Policy 19, 234-254.

6. Maxwell DG, Ahiadeke C, Levin C, Armar-Klemesu M, Zakariah S \& Lamptey GM (1999) Alternative food-security indicators: revisiting the frequency and severity of 'coping strategies'. Food Policy 24, 411-429.

7. Radimer KL, Olson CM, Greene JC, Campbell CC \& Habicht JP (1992) Understanding hunger and developing indicators to assess it in women and children. J Nutr Educ 24, 36S-45S.

8. Kendall A, Olson CM \& Frongillo EA Jr (1995) Validation of the Radimer/Cornell measures of hunger and food insecurity. J Nutr 125, 2793-2801.

9. Studdert LJ, Frongillo EA Jr \& Valois P (2001) Household food insecurity was prevalent in Java during Indonesia's economic crisis. J Nutr 131, 2685-2691.

10. Nnkwe N \& Yegammia C (2002) Prevalence of food insecurity among households with children in Coimbatore India. Nutr Res 22, 1009-1016.

11. Zalilah MS \& Ang M (2001) Assessment of food insecurity among low income households in Kuala Lumpur using the Radimer/Cornell food insecurity instrument $-\mathrm{a}$ validation study. Malays J Nutr 7, 15-32.

12. Sharif ZM \& Khor GL (2005) Obesity and household food insecurity: evidence from a sample of rural households in Malaysia. Eur J Clin Nutr 59, 1049-1058.

13. Wilde PE (2004) Differential response patterns affect foodsecurity prevalence estimates for households with and without children. J Nutr 134, 1910-1915.

14. Kennedy E (2003) Qualitative measures of food security in developing countries. In Proceedings of the International Scientific Symposium on 'Measurement and assessment of food deprivation and undernutrition', Rome, 26-28 June 2002. Rome: FAO; available at http://www.fao.org/docrep/ 005/Y4249E/y4249e0c.htm\#bm12

15. Coates J, Frongillo EA, Rogers BL, Webb P, Wilde PE \& Houser R (2006) Commonalities in the experience of household food insecurity across cultures: what are measures missing? J Nutr 136, 1438S-1448S.

16. Klouman E, Masenga EJ, Klepp KI, Sam NE, Nkya W \& Nkya C (1997) HIV and reproductive tract infections in a total village population in rural Kilimanjaro, Tanzania: women at increased risk. J Acquir Immune Defic Syndr Hum Retrovirol 14, 163-168.

17. Mmbaga EJ, Hussain A, Leyna GH, Klouman E, Masenga E, Sam N, Mnyika KS \& Klepp KI (2006) Incidence of HIV-1 infection and changes in prevalence of reproductive tract infections and sexual risk behaviours: a population-based longitudinal study in rural Tanzania. Afr J AIDS Res 5, 281-288.

18. King FS \& Burgess A (1993) Nutrition for Developing Countries, 2nd ed. Oxford: Oxford University Press.

19. Nunnaly JC \& Bernstein IH (1994) Psychometric Theory, 3rd ed., pp. 94-100. New York: McGraw Hill, Inc.

20. Frongillo EA \& Nanama S (2006) Development and validation of an experience-based measure of household food insecurity within and across seasons in Northern Burkina Faso. J Nutr 136, 1409S-1419S.

21. Frongillo EA Jr (1999) Validation of measures of food insecurity and hunger. $J$ Nutr 129, 506S-509S.

22. Carmines E \& Zeller R (1979) Reliability and Validity Assessments. Beverly Hills, CA: Sage.

23. Derrickson JP, Sakai M \& Anderson J (2001) Interpretations of the 'balanced meal' household food security indicator. J Nutr Educ 33, 155-160.

24. Welch KJ, Mock N \& Netrebenko O (1998) Measuring hunger in the Russian Federation using the Radimer/Cornell hunger scale. Bull World Health Organ 76, 143-148.

25. Schmidt FL \& Hunter JE (1977) Development of a general solution to the problem of validity generalization. $J A p p l$ Psychol 62, 529-540. 